\title{
A Equação de Condução de Calor Uni e Bidimensional: Solução Usando Transformada Integral e o Método da Separação de Variáveis
}

\section{The Uni and Bidimensional Heat Conduction Equation: Solution Using Integral Transform and the Method of Variable Separation}

\author{
Reynaldo D'Alessandro Neto \\ Universidade Federal de São Carlos, Sorocaba, SP \\ reynaldo.dalessandro@gmail.com \\ Antonio Luís Venezuela \\ Universidade Federal de São Carlos, Sorocaba, SP \\ alvenez@ufscar.br
}

\begin{abstract}
Resumo: As propriedades térmicas dos materiais são de grande importância para os projetos mecânicos, principalmente os que envolvem sistemas térmicos. A simulação e determinação do campo da temperatura pelo modelo matemático conhecido como equação do calor, auxilia na representação do comportamento térmico, isto é, nos fornece informações prévias de como a temperatura varia com a posição e o tempo em um sólido, e assim, poder caracterizar o material termicamente e saber as condições apropriadas a se impor ao objeto em estudo. O objetivo deste trabalho, é resolver a EDP que modela os processos de transporte de calor unidimensional e bidimensional em geometria retangular por meio da técnica da transformada integral clássica e separação de variáveis, respectivamente. Por fim, faz-se a análise dos modelos encontrados a partir da utilização de gráficos e tabelas de convergência.
\end{abstract}

Palavras-chave: transformada integral; método de Fourier; equação do calor.

\begin{abstract}
The thermal properties of materials are of great importance for mechanical projects, especially those involving thermal systems. The simulation and determining the mathematical model for the temperature field known as heat equation, assists in the representation of the thermal behavior, that is, it gives us prior information on how the temperature varies with the position and time in a solid, and so power thermally characterize the material and know the appropriate conditions to impose on the object under study. The objective of this work is to solve the EDP modeling the one-dimensional and two-dimensional heat transfer processes in rectangular geometry through the Classical Integral Transformation Technique and the Separation of Variables, respectively. Finally, we analyze the models found from the use of graphs and convergence tables
\end{abstract}

Key words: integral transformation; Fourier method; heat equation.

\section{Introdução}

Com o avanço tecnológico, os estudos que envolvem a transferência de calor ganham um grande destaque, já que a maioria dos processos industriais e de projetos de usinas nucleares e térmicas utilizam equipamentos de troca de calor como geradores de vapor, fornos, motores

Recebido em 29/08/2017 - Aceito em 28/11/2017

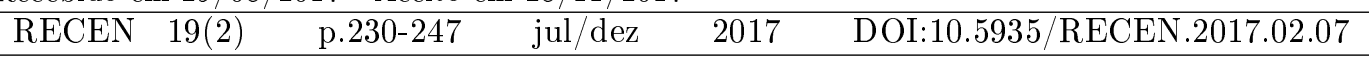


de calor, condensadores e outros. O mesmo acontece na área de produção de energia, que está em processo de expansão com projetos no controle do meio ambiente.

Existem outros processos em nosso dia-a-dia onde ocorre a transferência de calor, como os conversores catalíticos presentes nos motores de combustão interna dos automóveis, as unidades de refrigeração e ar-condicionado, os equipamentos eletrônicos, a refrigeração de motores elétricos, os transformadores e geradores elétricos, aquecimento e refrigeração de processos químicos, a minimização de perdas de calor em construções e aprimoramento de técnicas de isolamento térmico.

Com essa vasta gama de aplicações, vemos que os problemas relativos a transferência de calor aparecem como enormes desafios a se resolver. Assim, matemáticos, físicos e engenheiros estão constantemente confrontando com a necessidade de se maximizar e/ou minimizar taxas de transferência de calor, impulsionando um avanço rápido em várias tecnologias de aprimoramento, incluindo o uso de superfícies estendidas, agitadores e campos elétricos ou magnéticos externos [1] e [2]

A transferência de calor ocorre por condução, convecção e radiação, mas na maioria das vezes, por combinação das mesmas [3]. A maioria destes problemas são tratados a partir das Equações de Conservação de Energia Térmica, as quais são resolvidas utilizando técnicas numéricas, analíticas ou híbridas (analíticas-numéricas). A partir da década de 80 , as técnicas analíticas e/ou híbridas têm sido desenvolvidas e utilizadas na solução destas equações, devido a sua versatilidade no tratamento matemático/computacional.

No trabalho de [4] examina-se analiticamente a transferência de calor convectivo laminar forçado de um fluido newtoniano em um microcanal entre duas placas paralelas e para isso é utilizada a Técnica da Transformada Integral Generalizada. A teoria dos estresses térmicos com base na equação de condução de calor relacionada a uma derivada temporal de ordem maior que 2 é usada para investigar os estresses térmicos em um corpo cilíndrico infinito, cuja solução é obtida aplicando transformação integral de Laplace e Weber [5].

O objetivo deste trabalho, oriundo da dissertação de mestrado do Mestrado Profissional em Matemática em Rede Nacional - PROFMAT [6], é resolver a EDP que modela os processos de transporte de calor unidimensional e bidimensional em geometria retangular, as quais estão acopladas às condições de contornos de Dirichlet. O primeiro problema de valor de contorno está relacionado a uma barra feita de material condutor térmico e o segundo problema vinculado a uma placa também feita de um material condutor. Inicialmente resolveremos o problema unidimensional utilizando a Técnica de Transformada Integral Clássica e, finalmente, o problema bidimensional será resolvido via Técnica de Separação de Variáveis. Para cada uma das soluções analíticas obtidas serão comparadas com resultados analíticos provindos da literatura, além disso serão analisados os perfis de temperatura relativos à variável tempo, $t$, e às variáveis espaciais, $x$ e $y$.

Este trabalho procura mostrar uma técnica analítica, que abrange a utilização de procedimentos matemáticos para a resolução de conceitos físicos para a modelagem do problema em questão. Com isso, temos uma avaliação com maior precisão das propriedades térmicas dos materiais que se trabalham. O resultado analítico de Equações Diferenciais Parciais, fornece melhor subsídio para análises térmicas de materiais em problemas de condução de calor, dessa forma esse trabalho ratifica a importância de se propor uma solução analítica a um problema físico, deixando assim a resolução mais próxima da realidade do fenômeno físico em questão. 
Revista Ciências Exatas e Naturais, Vol.19, nº.2, Jul/Dez, 2017

\section{Modelagem Matemática}

A modelagem da equação do calor é desenvolvida, por um lado, a partir da Lei de Fourier e das equações do fluxo de calor e balanço de energia e, por outro lado, pelas equações da capacidade térmica e da massa específica [3] e [6]. Neste contexto, há a ocorrência da difusividade térmica $\alpha=k / c \rho$, que possui as dimensões $m^{2} / s$, sendo $k(W / m K)$ a condutividade térmica, $c(J / K)$ a capacidade térmica e $\rho\left(\mathrm{kg} / \mathrm{m}^{3}\right)$ a densidade.

Sejam $\Omega \subseteq \mathbb{R}^{r}, r \geqslant 1$, um aberto limitado de fronteira $\Gamma, \bar{\Omega}=\Omega \cup \Gamma, Q=\Omega \times(0,+\infty)$ e $\Sigma=\Gamma \times(0,+\infty)$. Procuramos uma função $u$, tal que $u \in C^{2}(\Omega \times(0,+\infty)) \cap C(\bar{\Omega} \times[0,+\infty))$, relativa ao problema de valor de contorno [7]:

$$
\begin{aligned}
\frac{\partial u}{\partial t} & =\alpha \Delta u, \text { em } \mathrm{Q}, \\
u & =\tilde{T}, \text { sobre } \Sigma, \\
u(v, 0) & =f(v), \text { em } \bar{\Omega},
\end{aligned}
$$

sendo $\Delta=\sum_{i=1}^{r} \frac{\partial^{2}}{\partial x_{i}^{2}}$ o laplaciano relativo às variáveis espaciais e $t$ à variável tempo (s). Como $u$ deve ser uma função diferenciável de classe $C^{2}(\Omega \times(0,+\infty))$, no mínimo, e também contínua, $C(\bar{\Omega} \times[0,+\infty))$, logo a função $f$ também deve ser continua, $C(\bar{\Omega})$.

A Equação (1) é a equação do calor, pois modela a distribuição da temperatura $u$ no domínio $\Omega$ e no instante $t$. A Equação (2) é a condiçãa de contorno de Dirichlet, a qual pode ser substituída pela condição de Neumann, $\frac{\partial u}{\partial \widehat{n}}=\tilde{T}$ sobre $\Sigma$, sendo $\widehat{n}$ o vetor unitário da normal exterior a $\Gamma$. A condição de Dirichlet expressa que o bordo $\Gamma$ de $\Omega$ se mantém a temperatura $\tilde{T}$. A condição de Neumann expressa que o fluxo de calor através do bordo $\Gamma$ é $\tilde{T}$. A Equação (3) é a condição inicial ou condição de Cauchy.

Para que exista solução do referido problema de valor de contorno, a função $f$ deve satisfazer a condição de compatibilidade: $f(v)=\tilde{T}, v \in \bar{\Omega}$.

Na sequência, expomos três problemas de valor de contorno, respectivamente, uni, bi e tridimensional, juntamente com o desenvolvimento matemático para se obter as soluções, isto é, a determinação do perfil de temperatura.

\subsection{Equação do calor unidimensional}

\section{Problema de valor de contorno unidimensional}

Considerando as Equações (1), (2) e (3), sejam $\Omega=\left(0, L_{x}\right) \subset \mathbb{R}, \Gamma=\left\{0, L_{x}\right\}, L_{x}>0$, assim temos uma barra de seção uniforme, $\bar{\Omega}$, com área muito pequena em relação ao comprimento $L_{x}$. Neste caso, não há troca de calor com o exterior através da superfície lateral da barra, sendo que os extremos correspondem ao bordo $\Gamma$ e estão mantidos à temperatura $\tilde{T}\left({ }^{\circ} C\right)$, conforme a condição de contorno (2). Procuramos uma função $u$, tal que $u \in C^{2}(\Omega \times(0,+\infty)) \cap C(\bar{\Omega} \times[0,+\infty))$, relativa ao problema de valor de contorno unidimensional [8]:

$$
\begin{aligned}
\frac{\partial u}{\partial t} & =\alpha \frac{\partial^{2} u}{\partial x^{2}}, \quad \text { em }\left(0, L_{x}\right) \times(0, \infty) \\
u(0, t) & =\tilde{T}, \quad t \in[0, \infty), \\
u\left(L_{x}, t\right) & =\tilde{T}, \quad t \in[0, \infty), \\
u(x, 0) & =f(x), \quad x \in\left[0, L_{x}\right] .
\end{aligned}
$$


D'ALESSANDRO NETO, R. e VENEZUELA, A. L.

Para resolver este problema, na sequência, utilizaremos a Técnica da Tranformada Integral Clássica-TTIC.

\section{Técnica da Tranformada Integral Clássica-TTIC}

A TTIC exige que as condições de contorno sejam homogêneas, mas isto não ocorre nas Equações (5) e (6). Com o objetivo de homogeneizá-las, aplicamos o seguinte filtro matemático:

$$
u(x, t)=u_{h}(x, t)+M(x),
$$

sendo que $M(x)$ é obtida a partir do seguinte problema de valor inicial:

$$
\begin{aligned}
\frac{d^{2}}{d x^{2}} M(x) & =0, \quad \text { em }\left(0, L_{x}\right) \\
M(0) & =\tilde{T} \\
M\left(L_{x}\right) & =\tilde{T} .
\end{aligned}
$$

Resolvendo a Equação (9) temos: $M(x)=c_{1} x+c_{2}$. As constantes $c_{1}$ e $c_{2}$ são determinadas usando as Equações (10) e (11), daí $c_{1}=0$ e $c_{2}=\tilde{T}$. Com isto, o filtro $M$ é dado por:

$$
M(x)=\tilde{T} .
$$

Substituindo a Equação (8) no problema inicial, Equações (4) - (7), e considerando a Equação (12), obtemos o problema de valor de contorno (com condições de contorno homogêneas):

$$
\begin{aligned}
\frac{\partial u_{h}}{\partial t} & =\alpha \frac{\partial^{2} u_{h}}{\partial x^{2}}, \quad \text { em }\left(0, L_{x}\right) \times(0, \infty) \\
u_{h}(0, t) & =0, \quad t \in[0, \infty) \\
u_{h}\left(L_{x}, t\right) & =0, \quad t \in[0, \infty) \\
u_{h}(x, 0) & =f(x)-\tilde{T}, \quad x \in\left[0, L_{x}\right]
\end{aligned}
$$

\section{Problema Auxiliar}

Seguindo a TTIC, o problema auxiliar (ou problema de autovalor) apropriado é dado por:

$$
\begin{aligned}
\frac{d^{2} \Psi_{n}(x)}{d x^{2}}+\lambda_{n}^{2} \Psi_{n}(x) & =0 \\
\Psi_{n}(0) & =0 \\
\Psi_{n}\left(L_{x}\right) & =0
\end{aligned}
$$

sendo $\Psi_{n}$ as autofunções associadas aos autovalores $\lambda_{n}$, sendo $n=0,1, \ldots$.

A solução deste problema auxiliar é dada por [9], a saber:

$$
\Psi_{n}(x)=\operatorname{sen}\left(\lambda_{n} x\right)
$$

Considerando a condição inicial, Equação (19), obtemos os autovalores:

$$
\lambda_{n}=\frac{n \pi}{L_{x}}
$$


Revista Ciências Exatas e Naturais, Vol.19, nº.2, Jul/Dez, 2017

A partir da propriedade de ortogonalização das autofunções $\Psi_{n}$ :

$$
\left\langle\Psi_{m}, \Psi_{n}\right\rangle=\int_{0}^{L_{x}} \operatorname{sen}\left(\lambda_{m} x\right) \operatorname{sen}\left(\lambda_{n} x\right) d x= \begin{cases}N_{n}, & m=n \\ 0, & m \neq n\end{cases}
$$

sendo $N_{n}$ dado por [9]:

$$
N_{n}=\int_{0}^{L_{x}} \Psi_{n}^{2} d x=\int_{0}^{L_{x}} \operatorname{sen}^{2}\left(\lambda_{n} x\right) d x=\frac{2}{L_{x}} .
$$

As autofunções normalizadas (ou núcleo) são dadas por, para $n=0,1,2, \ldots$ :

$$
\tilde{\Psi}_{n}(x)=\frac{\Psi_{n}(x)}{N_{n}^{1 / 2}} \quad \Longrightarrow \quad \tilde{\Psi}_{n}(x)=\sqrt{\frac{2}{L_{x}}} \operatorname{sen}\left(\lambda_{n} x\right)
$$

Logo, temos que:

$$
\left\langle\tilde{\Psi}_{m}, \tilde{\Psi}_{n}\right\rangle=\int_{0}^{L_{x}} \tilde{\Psi}_{m} \tilde{\Psi}_{n} d x=\delta_{m n}= \begin{cases}1, & m=n \\ 0, & m \neq n\end{cases}
$$

sendo $\delta_{m n}$ o delta de Kronecker.

\section{Par Transformada-Inversa}

O próximo passo da TTIC é determinar o par transformada-fórmula de inversão:

$$
\begin{aligned}
\bar{u}_{n}(t) & =\int_{0}^{L_{x}} \tilde{\Psi}_{n}(x) u_{h}(x, t) d x: \text { Transformada, } \\
u_{h}(x, t) & =\sum_{n=0}^{\infty} \tilde{\Psi}_{n}(x) \bar{u}_{n}(t): \text { Fórmula de inversão. }
\end{aligned}
$$

A partir da fórmula de inversão, temos:

$$
\frac{\partial u_{h}}{\partial t}=\sum_{m=0}^{\infty} \tilde{\Psi}_{m}(x) \bar{u}_{m}^{\prime}(t)
$$

e

$$
\frac{\partial^{2} u_{h}}{\partial x^{2}}=\sum_{m=0}^{\infty} \tilde{\Psi}_{m}^{\prime \prime}(x) \bar{u}_{m}(t) .
$$

Na Equação (13) multiplicamos ambos os membros por $\tilde{\Psi}_{n}(x)$ e aplicamos a integral definida no intervalo $\left[0, L_{x}\right]$ e nesta substituimos as Equações (24), (25) e (21), com isso obtemos:

$$
\sum_{m=0}^{\infty} \bar{u}_{m}^{\prime}(t) \int_{0}^{L_{x}} \tilde{\Psi}_{n}(x) \tilde{\Psi}_{m}(x) d x=\sum_{m=0}^{\infty}\left(-\lambda_{m}^{2} \alpha\right) \bar{u}_{m}(t) \int_{0}^{L_{x}} \tilde{\Psi}_{n}(x) \tilde{\Psi}_{m}(x) d x
$$


Logo, para $m, n=0,1,2, \ldots$, obtemos:

$$
\sum_{m=0}^{\infty} \delta_{m n} \bar{u}_{m}^{\prime}(t)=\sum_{m=0}^{\infty}\left(-\alpha \lambda_{m} \lambda_{n} \delta_{m n}\right) \bar{u}_{m}(t)
$$

As Equações (26) podem ser escritas na forma matricial e referem-se a um sistema infinito de Equações Diferenciais Ordinárias lineares homogêneas, ou seja:

$$
\left(\begin{array}{c}
\bar{u}_{0}^{\prime}(t) \\
\bar{u}_{1}^{\prime}(t) \\
\vdots \\
\bar{u}_{n}^{\prime}(t) \\
\vdots
\end{array}\right)=\left(\begin{array}{cccccc}
-\lambda_{0}^{2} \alpha & 0 & \ldots & 0 & 0 & \ldots \\
0 & -\lambda_{1}^{2} \alpha & \ldots & 0 & 0 & \ldots \\
\vdots & \vdots & \ldots & \vdots & \vdots & \ldots \\
0 & 0 & \ldots & 0 & -\lambda_{n}^{2} \alpha & \ldots \\
\vdots & \vdots & \ldots & \vdots & \vdots & \ldots
\end{array}\right)\left(\begin{array}{c}
\bar{u}_{0}(t) \\
\bar{u}_{1}(t) \\
\vdots \\
\bar{u}_{n}(t) \\
\vdots
\end{array}\right)
$$

Assim, para cada $n=0,1,2, \ldots$, o sistema acima reduz-se ao problema de valor inicial:

$$
\bar{u}_{n}^{\prime}(t)=-\alpha \lambda_{n}^{2} \bar{u}_{n}(t)
$$

cuja condição inicial é dada pela Transformada, Equação (22), no ponto $t=0$, isto é:

$$
\bar{u}_{n}(0)=\int_{0}^{L_{x}} \tilde{\Psi}_{n}(x)(f(x)-\tilde{T}) d x .
$$

A solução do problema dado pelas Equações (27) e (28) é:

$$
\bar{u}_{n}(t)=\bar{u}_{n}(0) e^{-\alpha \lambda_{n}^{2} t}
$$

Substituindo a Equação (20) e a Equação (29) na fórmula de inversão, Equação (23), obtemos:

$$
u_{h}(x, t)=\sum_{n=0}^{\infty} \sqrt{\frac{2}{L_{x}}} \operatorname{sen}\left(\lambda_{n} x\right) \bar{u}_{n}(0) e^{-\alpha \lambda_{n}^{2} t} .
$$

Substituindo as Equações (12) e (30) na Equação (8), temos:

$$
u(x, t)=\sum_{n=0}^{\infty} \sqrt{\frac{2}{L_{x}}} \operatorname{sen}\left(\lambda_{n} x\right) \bar{u}_{n}(0) e^{-\alpha \lambda_{n}^{2} t}+\tilde{T},
$$

sendo:

$$
\bar{u}_{n}(0)=\sqrt{\frac{2}{L_{x}}} \int_{0}^{L_{x}} \operatorname{sen}\left(\lambda_{n} x\right)(f(x)-\tilde{T}) d x .
$$

Portanto, a solução da equação do calor unidimensional, via TTIC, é dada por:

$$
u(x, t)=\frac{2}{L_{x}} \sum_{n=0}^{\infty} \operatorname{sen}\left(\lambda_{n} x\right) e^{-\alpha \lambda_{n}^{2} t} \int_{0}^{L_{x}} \operatorname{sen}\left(\lambda_{n} x\right)(f(x)-\tilde{T}) d x+\tilde{T} .
$$


Revista Ciências Exatas e Naturais, Vol.19, nº.2, Jul/Dez, 2017

\subsection{Equação do calor bidimensional}

\section{Problema de valor de contorno bidimensional}

Considerando as Equações (1), (2) e (3), sejam $\Omega=\left(0, L_{x}\right) \times\left(0, L_{y}\right) \subset \mathbb{R}^{2}, L_{x}>0$ e $L_{y}>0$, e o bordo $\Gamma$ formado pelas arestas do retângulo $\left[0, L_{x}\right] \times\left[\left(0, L_{y}\right]\right.$. Com isto, temos uma placa plana retangular de material homogêneo, $\bar{\Omega}$, onde não há troca de calor com o exterior através da superfície lateral da placa, sendo que os extremos correspondem ao bordo, $\Gamma$, e estão mantidos à temperatura constante igual a zero, $\tilde{T}=0\left({ }^{\circ} \mathrm{C}\right)$, conforme a condição de contorno (2).

Procuramos uma função $u$, tal que $u \in C^{2}(\Omega \times(0,+\infty)) \cap C(\bar{\Omega} \times[0,+\infty))$, relativa ao problema de valor de contorno bidimensional [8]:

$$
\begin{aligned}
\frac{\partial u}{\partial t} & =\alpha\left(\frac{\partial^{2} u}{\partial x^{2}}+\frac{\partial^{2} u}{\partial y^{2}}\right), \quad \operatorname{em} \Omega \times(0, \infty) \\
u(0, y, t) & =0, \quad y \in\left[0, L_{y}\right], \quad t \in[0, \infty), \\
u\left(L_{x}, y, t\right) & =0, \quad y \in\left[0, L_{y}\right], \quad t \in[0, \infty), \\
u(x, 0, t) & =0, \quad x \in\left[0, L_{x}\right], \quad t \in[0, \infty), \\
u\left(x, L_{y}, t\right) & =0, \quad x \in\left[0, L_{x}\right], \quad t \in[0, \infty), \\
u(x, y, 0) & =f(x, y), \quad(x, y) \in \bar{\Omega},
\end{aligned}
$$

\section{Separação de Variáveis}

Para a resolução do problema de condução de calor bidimensional com condições de contornos homogêneos, será utilizado o método da separação de variáveis. Para isso, tomamos a função $u$ definida da seguinte forma:

$$
u(x, y, t)=F(x) G(y) H(t),
$$

sendo $F, G$, funções diferenciáveis de classe $C^{2}(\Omega \times(0,+\infty))$, no mínimo, relativas às variáveis $x$ e $y$, respectivamente, e $H$ uma função diferenciável de classe $C^{1}(\Omega \times(0,+\infty))$, no mínimo, relativa a variável $t$. Logo, temos que $\frac{\partial u}{\partial t}=F(x) G(y) \frac{d H(t)}{d t}, \frac{\partial^{2} u}{\partial^{2} x}=\frac{d^{2} F(x)}{d^{2} x} G(y) H(t)$ e $\frac{\partial^{2} u}{\partial^{2} y}=F(x) \frac{d^{2} G(y)}{d^{2} y} H(t)$. Substituindo estas expressões na Equação (33), obtemos:

$$
F(x) G(y) H_{t}(t)=\alpha\left(F_{x x}(x) G(y) H(t)+F(x) G_{y y} H(t)\right),
$$

onde considerando $H_{t}(t)=\frac{d H(t)}{d t}, F_{x x}(x)=\frac{d^{2} F(x)}{d^{2} x}$ e $G_{y y}(y)=\frac{d^{2} G(y)}{d^{2} y}$.

Na expressão acima, dividimos ambos lados por $\alpha F(x) G(y) H(t) \neq 0$, para $x \in\left[0, L_{x}\right], y \in$ $\left[0, L_{y}\right]$ e $t \in[0,+\infty]$, daí obtemos:

$$
\frac{1}{\alpha} \frac{H_{t}(t)}{H(t)}=\frac{F_{x x}(x)}{F(x)}+\frac{G_{y y}(y)}{G(y)} .
$$

O lado esquerdo da Equação (40) depende somente de $t$ e, o lado direito, depende das variáveis $x$ e $y$. Desta forma, podemos representar cada lado desta expressão pela constante, $-\sigma^{2}$, assim temos que:

$$
\begin{aligned}
\frac{1}{\alpha} \frac{H_{t}(t)}{H(t)} & =-\sigma^{2}, \quad \mathrm{e} \\
\frac{F_{x x}(x)}{F(x)}+\frac{G_{y y}(y)}{G(y)} & =-\sigma^{2} .
\end{aligned}
$$


Na Equação (42) fazemos:

$$
\frac{F_{x x}(x)}{F(x)}=-\frac{G_{y y}(y)}{G(y)}-\sigma^{2},
$$

e analogamente ao que fizemos acima, cada lado da Equação (43) pode ser igualado à constante " $-\xi^{2}$ ", já que o lado esquerdo depende somente da variável $x$ e o lado direito depende da variável $y$. Desta forma, temos:

$$
\begin{aligned}
\frac{F_{x x}(x)}{F(x)} & =-\xi^{2}, \quad \mathrm{e} \\
-\frac{G_{y y}(y)}{G(y)}-\sigma^{2} & =-\xi^{2} .
\end{aligned}
$$

Considerando $\eta^{2}=\sigma^{2}-\xi^{2}$, temos a seguinte expressão:

$$
\frac{G_{y y}(y)}{G(y)}=-\eta^{2}
$$

Aplicamos as condições de contorno, Equações (34) até (38), na Equação (39), daí, a partir das Equações (44) e (45), temos os seguintes problemas de valor inicial:

$$
\begin{aligned}
& \left\{\begin{array}{l}
F_{x x}(x)+\xi^{2} F(x)=0 \\
F(0)=F\left(L_{x}\right)=0
\end{array}\right. \\
& \left\{\begin{array}{l}
G_{y y}(y)+\eta^{2} G(y)=0 \\
G(0)=G\left(L_{y}\right)=0
\end{array}\right.
\end{aligned}
$$

As autofunções $F_{m}$ relativas aos autovalores $\xi_{m}$ do problema de valor inicial (46) e as autofunções $G_{n}$ relativas aos autovalores $\eta_{n}$ do problema de valor inicial (47), conforme [9], respectivamente, são dadas, para $m, n=0,1,2, \ldots$ :

$$
\begin{array}{r}
\left\{\begin{array}{l}
F_{m}(x)=\operatorname{sen}\left(\xi_{m} x\right) \\
\xi_{m}=\frac{m \pi}{L_{x}}
\end{array}\right. \\
\left\{\begin{array}{l}
G_{n}(y)=\operatorname{sen}\left(\eta_{n} y\right) \\
\eta_{n}=\frac{n \pi}{L_{y}}
\end{array}\right.
\end{array}
$$

A partir da Equação (41) temos a equação diferencial ordinária homogênea $H_{t}(t)+$ $\sigma^{2} \alpha H(t)=0$, cuja solução geral é dada por $H_{m n}(t)=c_{0} e^{-\sigma_{m n}^{2} \alpha t}$, sendo $\sigma_{m n}^{2}=\xi_{m}^{2}+\eta_{n}^{2}$, e sem perda da generalidade, tomamos $c_{0}=1$, logo:

$$
H_{m n}(t)=e^{-\sigma_{m n}^{2} \alpha t}
$$

Pelo princípio da superposição, temos que:

$$
u(x, y, t)=\sum_{m=0}^{\infty} \sum_{n=0}^{\infty} A_{m n} u_{m n}(x, y, t)
$$

sendo $u_{m n}(x, y, t)=F_{m}(x) G_{n}(y) H_{m n}(t)$.

A seguir determinaremos os coeficientes $A_{m n}$ e o perfil de temperatura $u$. 
Revista Ciências Exatas e Naturais, Vol.19, nº.2, Jul/Dez, 2017

(a) Condição inicial para $t=0$

Pela condição inicial, Equação (34), e considerando a Equação (50), tem-se $H_{m n}(0)=1$, logo, a Equação (51) é dada por:

$$
f(x, y)=\sum_{m=0}^{\infty} \sum_{n=0}^{\infty} A_{m n} F_{m}(x) G_{n}(y)
$$

\section{(b) Coeficientes $A_{m n}$}

Sendo $u_{1} \in C(\bar{\Omega} \times\{0\})$ e $u_{2} \in C^{2}(\Omega \times\{0\})$, definimos o produto interno:

$$
\left\langle u_{1}, u_{2}\right\rangle=\int_{0}^{L_{x}} \int_{0}^{L_{y}} u_{1} u_{2} d y d x
$$

Tomando $u_{1}=u_{1}(x, y, 0)=f(x, y)$ e $u_{2}=u_{2}(x, y, 0)=F_{p}(x) G_{q}(y), p, q=0,1,2, \ldots$, e pela Equação (52), juntamente com as propriedades do produto interno, temos:

$$
\left\langle f(x, y), F_{p}(x) G_{q}(y)\right\rangle=\sum_{m=0}^{\infty} \sum_{n=0}^{\infty} A_{m n}\left\langle F_{m}(x) G_{n}(y), F_{p}(x) G_{q}(y)\right\rangle .
$$

Pela propriedade de ortogonalidade [10], temos que:

$$
\left\langle F_{m}(x) G_{n}(y), F_{p}(x) G_{q}(y)\right\rangle= \begin{cases}N_{m n}, & m=p \text { e } n=q \\ 0, & m \neq p \text { e } n \neq q\end{cases}
$$

sendo $N_{m n}=\int_{0}^{L_{x}} \int_{0}^{L_{y}} F_{m}^{2} G_{n}^{2} d y d x$ e considerando as autofunções, Equações (48) e (49), obtemos:

$$
\left\langle f(x, y), F_{m}(x) G_{n}(y)\right\rangle=A_{m n} \frac{L_{x} L_{y}}{4} .
$$

Logo, para $m, n=0,1,2, \ldots$ :

$$
A_{m n}=\frac{4}{L_{x} L_{y}} \int_{0}^{L x} \int_{0}^{L y} f(x, y) F_{m}(x) G_{n}(y) d y d x .
$$

(c) Perfil de temperatura $u=u(x, y, t)$

Na Equação (51) substituímos as Equações (48), (49) e (50) e, com isso, determinamos a solução da equação do calor bidimensional, ou seja:

$$
u(x, y, t)=\sum_{m=0}^{\infty} \sum_{n=0}^{\infty} A_{m n} e^{-\alpha \pi^{2}\left(\left(\frac{m}{L_{x}}\right)^{2}+\left(\frac{n}{L_{y}}\right)^{2}\right) t} \operatorname{sen}\left(\frac{m x \pi}{L_{x}}\right) \operatorname{sen}\left(\frac{n y \pi}{L_{y}}\right) .
$$


D'ALESSANDRO NETO, R. e VENEZUELA, A. L.

\subsection{Equação do calor tridimensional}

\section{Problema de valor de contorno tridimensional}

No objetivo deste trabalho propomos determinar as soluções dos problemas de condição de valor de contorno uni e bidimensional, contudo, estendemos o problema de condução de calor da placa plana para um sólido e para este não será realizado a análise dos resultados.

Considerando as Equações (1), (2) e (3), sejam $\Omega=\left(0, L_{x}\right) \times\left(0, L_{y}\right) \times\left(0, L_{z}\right) \subset \mathbb{R}^{3}$, $L_{x}>0, L_{y}>0$ e $L_{z}>0$, e o bordo $\Gamma$ formado pelas faces do paralelogramo regular $\left[0, L_{x}\right] \times\left[0, L_{y}\right] \times\left[0, L_{z}\right]$. Desta forma, temos um sólido de material homogêneo, $\bar{\Omega}$, onde não há troca de calor com o exterior através da superfície lateral deste sólido, sendo que os extremos correspondem ao bordo, $\Gamma$, e estão mantidos à temperatura constante igual a zero, $\tilde{T}=0\left({ }^{\circ} \mathrm{C}\right)$, conforme a condição de contorno $(2)$.

Procuramos uma função $u$, tal que $u \in C^{2}(\Omega \times(0,+\infty)) \cap C(\bar{\Omega} \times[0,+\infty))$, relativa ao problema de valor de contorno tridimensional [8]:

$$
\begin{aligned}
\frac{\partial u}{\partial t} & =\alpha\left(\frac{\partial^{2} u}{\partial x^{2}}+\frac{\partial^{2} u}{\partial y^{2}}+\frac{\partial^{2} u}{\partial z^{2}}\right), \quad \text { em } \Omega \times(0, \infty), \\
u(0, y, z, t) & =0, \quad y \in\left[0, L_{y}\right], \quad z \in\left[0, L_{z}\right] \text { e } t \in[0, \infty), \\
u\left(L_{x}, y, z, t\right) & =0, \quad y \in\left[0, L_{y}\right], \quad z \in\left[0, L_{z}\right] \text { e } t \in[0, \infty), \\
u(x, 0, z, t) & =0, \quad x \in\left[0, L_{x}\right], \quad z \in\left[0, L_{z}\right] \text { e } t \in[0, \infty), \\
u\left(x, L_{y}, z, t\right) & =0, \quad x \in\left[0, L_{x}\right], \quad z \in\left[0, L_{z}\right] \text { e } t \in[0, \infty), \\
u(x, y, 0, t) & =0, \quad x \in\left[0, L_{x}\right], \quad y \in\left[0, L_{y}\right] \text { e } t \in[0, \infty), \\
u\left(x, y, L_{z}, t\right) & =0, \quad x \in\left[0, L_{x}\right], \quad y \in\left[0, L_{y}\right] \text { e } t \in[0, \infty), \\
u(x, y, z, 0) & =f(x, y, z), \quad(x, y, z) \in \bar{\Omega} .
\end{aligned}
$$

A solução do problema de valor de contorno relativo à equação do calor tridimensional é obtida de forma análoga ao procedimento realizado para a equação do calor bidimensional, ou seja, utilizando a separação de variáveis, $u(x, y, z, t)=F(x) G(y) H(z) V(t)$, temos:

$$
\begin{aligned}
u(x, y, z, t) & =\sum_{m, n, k=0}^{\infty} A_{m n k} e^{-\alpha \pi^{2}\left(\left(\frac{m}{L_{x}}\right)^{2}+\left(\frac{n}{L_{y}}\right)^{2}+\left(\frac{k}{L_{z}}\right)^{2}\right) t} \operatorname{sen}\left(\frac{m x \pi}{L_{x}}\right) \operatorname{sen}\left(\frac{n y \pi}{L_{y}}\right) \operatorname{sen}\left(\frac{k z \pi}{L_{z}}\right), \\
A_{m n k} & =\frac{4}{L_{x} L_{y} L_{z}} \int_{0}^{L x} \int_{0}^{L y} \int_{0}^{L z} f(x, y, z) F_{m}(x) G_{n}(y) H_{k}(z) d z d y d x,
\end{aligned}
$$

sendo $F_{m}(x)=\operatorname{sen}\left(\frac{m x \pi}{L_{x}}\right), G_{n}(y)=\operatorname{sen}\left(\frac{n y \pi}{L_{y}}\right)$ e $H_{k}(z)=\operatorname{sen}\left(\frac{k z \pi}{L_{z}}\right)$.

\section{Análise dos resultados}

Nesta seção serão analisados a solução, via TTIC, da equação do calor unidimensional, Equação (32), e a solução, via separação de variáveis, da equação do calor bidimensional, Equação (53). Para cada caso, será determinada a ordem de truncamento, $N$, com erro relativo global previamente definido, bem como a análise qualitativa (ou coerência dos resultados provindos da solução exata relativamente ao modelo físico) por intermédio de gráficos gerados pelo software SciDaVis [11]. Os resultados do perfil de temperatura relativos à equação do calor unidimensional e bidimensional foram gerados utilizando o sistema algébrico computacional SAGe [12, 13]. 
Revista Ciências Exatas e Naturais, Vol.19, nº.2, Jul/Dez, 2017

Na Tabela (1) temos os resultados do perfil de temperatura relacionados à equação do calor unidimensional e na Tabela (2) representamos os resultados do perfil de temperatura relacionados à equação do calor bidimensional, onde, em ambos os casos, estabelecemos a ordem de truncamento.

Relativamente à equação de calor unidimensional, nas Figuras (1) e (2) são apresentados os resultados do perfil de temperatura, respectivamente, em função da coordenada tempo $t$ e $x$. Na Figura (3) temos a temperatura representada no espaço tridimensional.

Nas Figuras (4) e (5) temos os perfis de temperatura, respectivamente, em função da coordenada tempo $t$ e $x$, referentes à equação do calor bidimensional. Na Figura (7) temos a temperatura representada no espaço tridimensional, onde fixamos uma das variáveis espaciais.

\subsection{Equação do Calor Unidimensional - Solução via TTIC}

Para esta análise, estabelecemos os seguintes parâmetros iniciais: $L_{x}=0,06 m, \alpha=$ $1,1410^{-4} \mathrm{~m}^{2} / \mathrm{s}$ (cobre), $f(x)=100^{\circ} \mathrm{C}, x \in\left[0 ; L_{x}\right], \tilde{T}=0^{\circ} C$ e $t(s) \in[0 ; 16]$. O tempo máximo foi considerado $16 \mathrm{~s}$, pois, para $t>16 \mathrm{~s}$, o perfil de temperatura $u$ tende assintoticamente a zero. Os valores da temperatura foram tomados com 3 casas decimais, onde consideramos esta a precisão do instrumento de medição de temperatura.

Tabela 1. (Equação do calor unidimensional) Análise da convergência da temperatura $u$, na posição $x=0,01 m$ em função da coordenada $t$.

\begin{tabular}{rrrrr}
\hline \multicolumn{5}{c}{$\boldsymbol{u}\left({ }^{\circ} C\right)$} \\
\hline $\boldsymbol{t}(\mathbf{s})$ & \multicolumn{1}{c}{$\mathbf{N}$} \\
\hline $\mathbf{2 , 4}$ & \multicolumn{1}{c}{$\mathbf{2}$} & \multicolumn{1}{c}{$\mathbf{3}$} & $\mathbf{4}$ \\
$\mathbf{3 , 2}$ & 14,474 & 18,584 & 18,624 & 18,624 \\
$\mathbf{4 , 0}$ & 11,271 & 11,271 & 14,477 & 14,477 \\
$\mathbf{4 , 8}$ & 8,778 & 8,778 & 8,778 & 11,271 \\
$\mathbf{5 , 6}$ & 6,836 & 6,836 & 6,836 & 6,836 \\
$\mathbf{6 , 4}$ & 5,324 & 5,324 & 5,324 & 5,324 \\
$\mathbf{7 , 2}$ & 4,146 & 4,146 & 4,146 & 4,146 \\
$\mathbf{8 , 0}$ & 3,229 & 3,229 & 3,229 & 3,229 \\
$\mathbf{8 , 8}$ & 2,514 & 2,514 & 2,514 & 2,514 \\
$\mathbf{9 , 6}$ & 1,958 & 1,958 & 1,958 & 1,958 \\
$\mathbf{1 0 , 4}$ & 1,525 & 1,525 & 1,525 & 1,525 \\
$\mathbf{1 1 , 2}$ & 1,188 & 1,188 & 1,188 & 1,188 \\
$\mathbf{1 2 , 0}$ & 0,925 & 0,925 & 0,925 & 0,925 \\
$\mathbf{1 2 , 8}$ & 0,720 & 0,720 & 0,720 & 0,720 \\
$\mathbf{1 3 , 6}$ & 0,561 & 0,561 & 0,561 & 0,561 \\
$\mathbf{1 4 , 4}$ & 0,437 & 0,437 & 0,437 & 0,437 \\
$\mathbf{1 5 , 2}$ & 0,340 & 0,340 & 0,340 & 0,340 \\
$\mathbf{1 6 , 0}$ & 0,265 & 0,265 & 0,265 & 0,265 \\
\hline
\end{tabular}

A Tabela (1) apresenta resultados para a temperatura, em função da variável $t$, para vários truncamentos $N$, relacionados à equação do calor unidimensional, onde fixamos $x=$ $0,01 \mathrm{~m}$. Para esta análise, podemos tomar qualquer valor de $x, x \in[0,00 ; 0,06]$, que teremos 
as mesmas ordens de truncamento. Para verificar a ordem de truncamento, fixamos uma linha, por exemplo, em $t=2,4 s$, e verificamos que, para $N=1$ temos $u=18,624$, para $N=2$ temos $u=18,584$, para $N=3$ temos $u=18,624$ e para $N \geq 4$ os valores $u=18,624$ se repetem. Para $t \geq 4,0 s$, a convergência de $u$ ocorre para $N=1$. Desta forma, consideramos a maior ordem de truncamento, assim $N=3$.

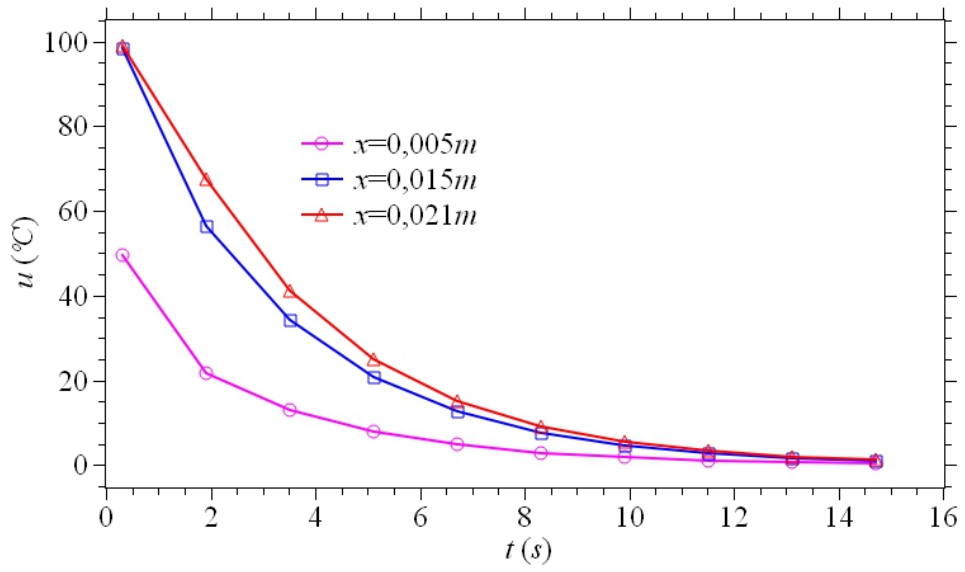

Figura 1. (Equação do calor unidimensional) Perfil de temperatura em função do tempo $t$, para as posições $x=0,005 m, x=0,015 m$ e $x=0,021 m$. Fonte: Próprio autor.

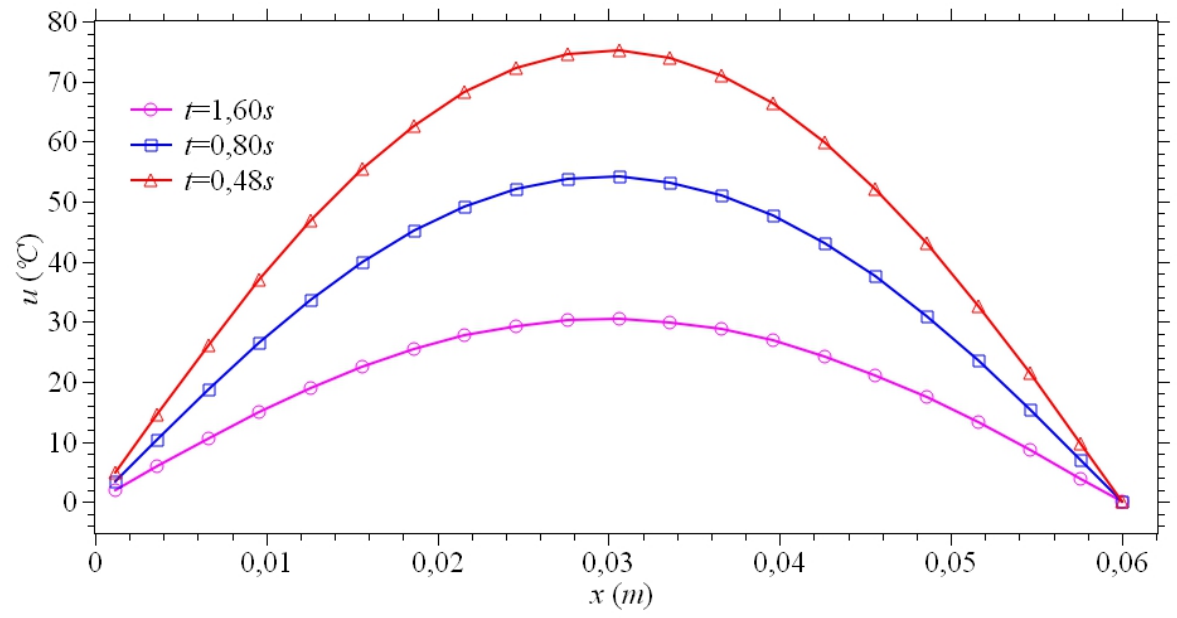

Figura 2. (Equação do calor unidimensional) Perfil de temperatura em função da posição $x$, para os tempos $t=0,48 s, t=0,80 \mathrm{~s}$ e $t=1,60 \mathrm{~s}$. Fonte: Próprio autor.

Na Figura (1) são apresentados os perfis da temperatura $u$ em função do tempo $t$, fixando $x=0,005 m, x=0,015 m$ e $x=0,021 \mathrm{~m}$. Vemos que, conforme o tempo aumenta a temperatura decresce, até atingir o valor mínimo igual a $\tilde{T}=0^{\circ} \mathrm{C}$. Aumentando a posição $x$, a temperatura $u$ também aumenta e isto ocorre até a posição $x=0,03 m$, e daí, a partir 
Revista Ciências Exatas e Naturais, Vol.19, nº.2, Jul/Dez, 2017

deste ponto, a temperatura começa à diminuir (ver Figura (2) ). Essa evolução está de acordo com a teoria física apresentada, ou seja, quando o material condutor cede calor, ocorre a diminuição da temperatura.

Temos na Figura (2) os perfis da temperatura $u$ em função da posição $x$, fixando $t=$ $0,48 s, t=0,80 \mathrm{~s}$ e $t=1,60 \mathrm{~s}$. Nas posição $x=0,00 \mathrm{~m}$ e $x=0,06 \mathrm{~m}$ a temperatura inicial é $\tilde{T}=0^{\circ} \mathrm{C}$ e, quando $t=0 \mathrm{~s}$ tem-se uma temperatura uniforme de $100^{\circ} \mathrm{C}$ em toda extensão da barra. Fixando o tempo, para $t>0$, observamos que, conforme se aumenta $x$ até a posição $x=0,03 \mathrm{~m}$ a temperatura cresce até $100^{\circ} \mathrm{C}$ e, a partir deste ponto até $x=0,06 \mathrm{~m}$, ocorre a diminuição da temperatura voltando para $\tilde{T}=0^{\circ} C$. Fixando uma posição $x$ vemos que, se aumentamos o tempo $t$, a temperatura diminui. Este processo está de acordo como fenômeno físico que envolve transporte de calor em uma barra.

Os efeitos físicos observados nas Figuras (1) e (2) podem ser vistos na Figura (3), que refere-se a representação gráfica do perfil de temperatura $u=u(x, t)$. A solução da equação do calor unidimensional, provinda do trabalho de [14], é equivalente a Equação (32).

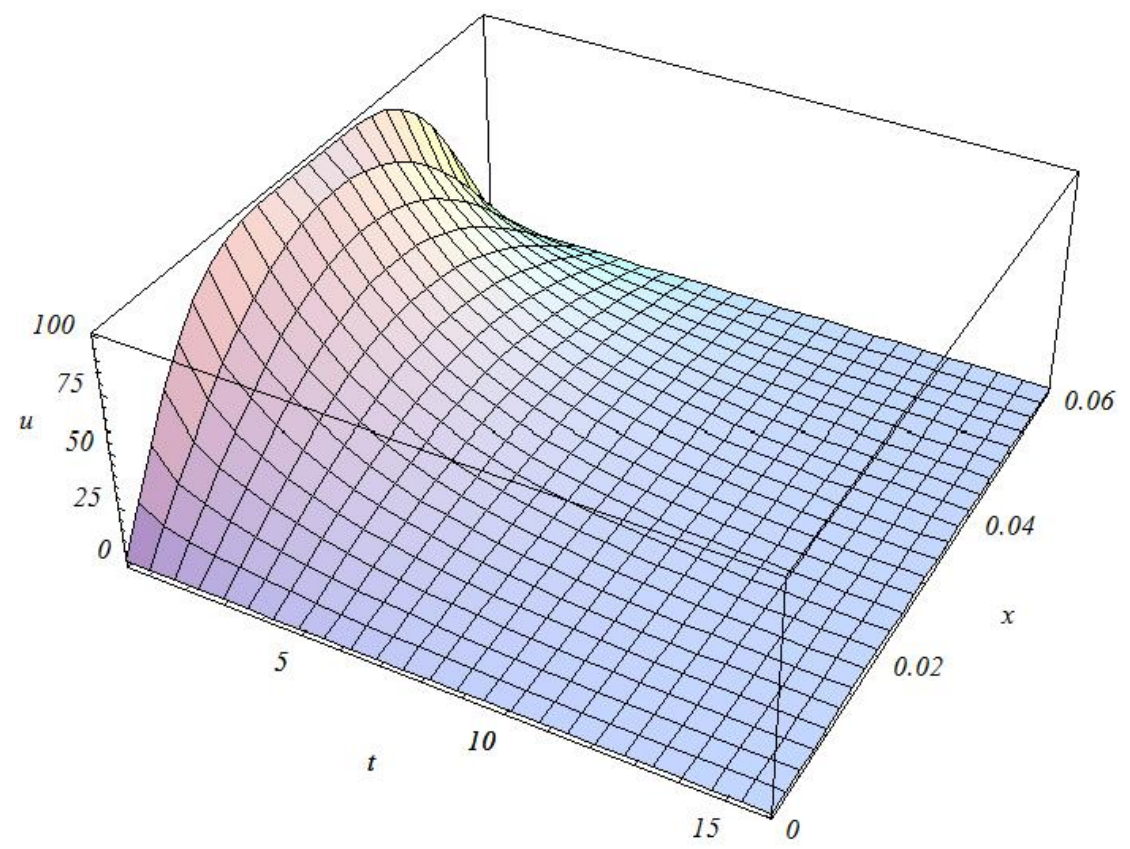

Figura 3. (Equação do calor unidimensional) Representação gráfica tridimensional do perfil de temperatura $u=u(x, t)$, considerando o domínio $(x, t) \in[0,00 ; 0,06] \times[0 ; 16]$. Fonte: Próprio autor.

\subsection{Equação do Calor Bidimensional - Solução via Separação de Variáveis}

Para esta análise, estabelecemos os seguintes parâmetros iniciais: $L_{x}=0,06 \mathrm{~m}, L_{y}=$ $0.10 m, \alpha=1,1410^{-4} m^{2} / s$ (cobre), $f(x, y)=100^{\circ} C, x \in\left[0 ; L_{x}\right], y \in\left[0 ; L_{y}\right]$ e $t \in[0 ; 16]$. O tempo máximo foi considerado $16 s$ e os valores da temperatura foram tomados com 3 casas decimais, conforme justificativa feita na seção anterior. 
D'ALESSANDRO NETO, R. e VENEZUELA, A. L.

Tabela 2. (Equação do calor bidimensional) Análise da convergência da temperatura u, na posição $x=0,01 m$ e $y=0,01 m$ em função da coordenada $t$.

\begin{tabular}{ccccccc}
\hline \multicolumn{7}{c}{$\boldsymbol{u}\left({ }^{\circ} C\right)$} \\
\hline \multicolumn{7}{c}{$\boldsymbol{N}$} \\
\hline $\mathbf{t}(\mathrm{s})$ & $\mathbf{1}$ & $\mathbf{2}$ & $\mathbf{3}$ & $\mathbf{4}$ & $\mathbf{5}$ & $\mathbf{6}$ \\
\hline $\mathbf{1 . 6}$ & 7.842 & 7.842 & 9.614 & 9.614 & 9.683 & 9.683 \\
$\mathbf{2 , 4}$ & 5,582 & 5,582 & 6,156 & 6,156 & 6,162 & 6,162 \\
$\mathbf{3 , 2}$ & 3,973 & 3,973 & 4,168 & 4,168 & 4,169 & 4,169 \\
$\mathbf{4 , 0}$ & 2,827 & 2,827 & 2,895 & 2,895 & 2,895 & 2,895 \\
$\mathbf{4 , 8}$ & 2,012 & 2,012 & 2,036 & 2,036 & 2,036 & 2,036 \\
$\mathbf{5 , 6}$ & 1,432 & 1,432 & 1,440 & 1,440 & 1,440 & 1,440 \\
$\mathbf{6 , 4}$ & 1,019 & 1,019 & 1,022 & 1,022 & 1,022 & 1,022 \\
$\mathbf{7 , 2}$ & 0,726 & 0,726 & 0,727 & 0,727 & 0,727 & 0,727 \\
$\mathbf{8 , 0}$ & 0,516 & 0,516 & 0,517 & 0,517 & 0,517 & 0,517 \\
$\mathbf{8 , 8}$ & 0,368 & 0,368 & 0,368 & 0,368 & 0,368 & 0,368 \\
$\mathbf{9 , 6}$ & 0,262 & 0,262 & 0,262 & 0,262 & 0,262 & 0,262 \\
$\mathbf{1 0 , 4}$ & 0,186 & 0,186 & 0,186 & 0,186 & 0,186 & 0,186 \\
$\mathbf{1 1 , 2}$ & 0,133 & 0,133 & 0,133 & 0,133 & 0,133 & 0,133 \\
$\mathbf{1 2 , 0}$ & 0,094 & 0,094 & 0,094 & 0,094 & 0,094 & 0,094 \\
$\mathbf{1 2 , 8}$ & 0,067 & 0,067 & 0,067 & 0,067 & 0,067 & 0,067 \\
$\mathbf{1 3 , 6}$ & 0,048 & 0,048 & 0,048 & 0,048 & 0,048 & 0,048 \\
$\mathbf{1 4 , 4}$ & 0,034 & 0,034 & 0,034 & 0,034 & 0,034 & 0,034 \\
$\mathbf{1 5 , 2}$ & 0,024 & 0,024 & 0,024 & 0,024 & 0,024 & 0,024 \\
$\mathbf{1 6 , 0}$ & 0,017 & 0,017 & 0,017 & 0,017 & 0,017 & 0,017 \\
\hline & & & & & & \\
\hline
\end{tabular}

A Tabela (2) apresenta resultados para a temperatura $u$, em função da variável $t$, para vários truncamentos $N$, relacionados à equação do calor bidimensional, onde fixamos $x=$ $0,01 \mathrm{~m}$ e $y=0,01 \mathrm{~m}$. Para esta análise, podemos tomar qualquer valor de $x$ e $y, x \in$ $[0,00 ; 0,06]$ e $y \in[0,00 ; 0,10]$, que teremos as mesmas ordens de truncamento. Para verificar a ordem de truncamento, fixamos uma linha, por exemplo, em $t=1,6 \mathrm{~s}$, e verificamos que, para $N=1$ temos $u=7,842$, para $N=2$ temos $u=7,842$, para $N=3$ temos $u=9,614$, para $N=4$ temos $u=9,614$, para $N=5$ temos $u=9,683$ e para $N \geq 6$ os valores $u=9,683$ se repetem. Para $t \geq 8,8 s$, a convergência de $u$ ocorre para $N=1$. Desta forma, consideramos a maior ordem de truncamento, assim $N=5$.

Na Figura (4) são apresentados os perfis da temperatura $u$ em função do tempo $t, t \in$ $[0 ; 16]$, para $y=0,01 \mathrm{~m}, y=0,04 \mathrm{~m}$ e $y=0,08 \mathrm{~m}$, fixando $x=0,01 \mathrm{~m}$. Vemos que, conforme o tempo cresce, a temperatura decresce, até atingir o valor mínimo igual a $\tilde{T}=0^{\circ} \mathrm{C}$. Aumentando a posição $y$, fixando $x$, a temperatura $u$ também aumenta. Essa evolução está de acordo com a teoria física apresentada, ou seja, quando o material condutor cede calor, ocorre a diminuição da temperatura.

Temos na Figura (5) os perfis da temperatura $u$ em função da posição $x, x \in[0,00 ; 0,06]$, para $y=0,01 \mathrm{~m}, y=0,04 \mathrm{~m}$ e $y=0,08 \mathrm{~m}$, fixando $t=1,6 \mathrm{~s}$. Nas posição $x=0,00 \mathrm{~m}$ e $x=0,06 \mathrm{~m}$ a temperatura inicial é $\tilde{T}=0^{\circ} \mathrm{C}$ e, quando $t=0 \mathrm{~s}$ tem-se uma temperatura uniforme de $100^{\circ} \mathrm{C}$ em toda extensão da barra. Fixando a posição $x$, observamos que, conforme se aumenta $x$ até a posição $x=0,03 \mathrm{~m}$ a temperatura cresce até $100^{\circ} \mathrm{C}$ e, a partir deste ponto até $x=0,06 \mathrm{~m}$, ocorre a diminuição da temperatura voltando para $\tilde{T}=0^{\circ} \mathrm{C}$. Fixando uma posição $x$ vemos que, se aumentamos o tempo $t$, a temperatura diminui. Este processo está de acordo como fenômeno fisico que envolve transporte de calor em uma barra. 
Revista Ciências Exatas e Naturais, Vol.19, nº.2, Jul/Dez, 2017

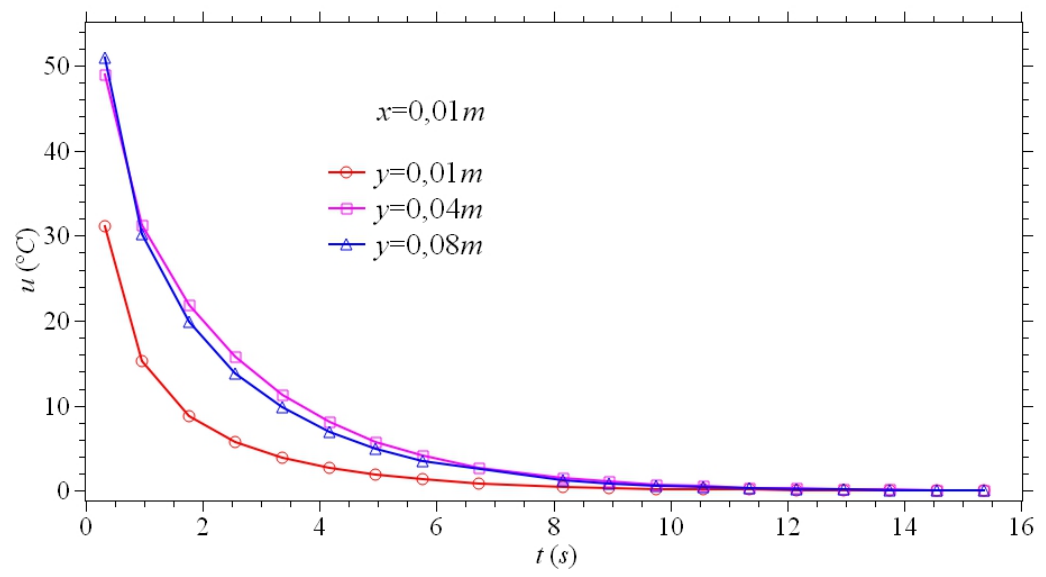

Figura 4. (Equação do calor bidimensional) Perfil de temperatura em função do tempo $t$, para as posições $y=0,01 m, y=0,04 m$ e $y=0,08 m$, fixando $x=0,01 m$. Fonte: Próprio autor.

Na Figura (6) mostramos os perfis da temperatura $u$ em função da posição $y, y \in$ $[0,00 ; 0,10]$, para $t=0,1,6 s, t=3,2 s$ e $t=4,8 s$, fixando $x=0,012 \mathrm{~m}$. Nas posições $y=0,00 \mathrm{~m}$ e $y=0,1 \mathrm{~m}$ a temperatura inicial é $\tilde{T}=0^{\circ} \mathrm{C}$ e, quando $t=0 \mathrm{~s}$ tem-se uma temperatura uniforme de $100^{\circ} \mathrm{C}$ em toda extensão da placa. Variando a posição $y$, observamos que, conforme se aumenta $t$ até a posição $y=0,05 \mathrm{~m}$ a temperatura cresce até $100^{\circ} \mathrm{C}$ e, a partir deste ponto até $y=0,1 \mathrm{~m}$, ocorre a diminuição da temperatura voltando para $\tilde{T}=0^{\circ} \mathrm{C}$. A oscilação térmica também é facilmente observada, como por exemplo, escolhendo arbitrariamente $x=0,012$ (poderia ser qualquer outro valor de $x$, pois o comportamento oscilatório é por toda placa, havendo apenas a variação da amplitude da temperatura), e com a variação de $y$, o perfil ondulatório é visto no gráfico. Por fim, o decaimento da temperatura ao longo de $y$ é esperado devido ao resfriamento que ocorreu nas extremidades da placa e que estão contempladas nas condições de contorno da Equação do Calor Bidimensional.

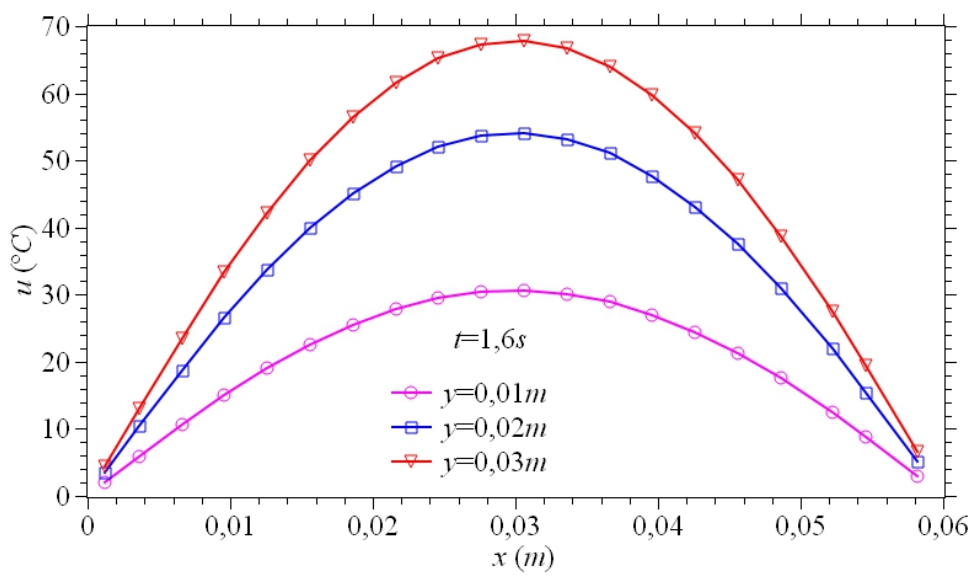

Figura 5. (Equação do calor bidimensional) Perfil de temperatura em função da coordenada $x$, para as posições $y=0,01 m, y=0,02 m$ e $y=0,03 m$, fixando $t=1,6 s$. Fonte: Próprio autor. 
D'ALESSANDRO NETO, R. e VENEZUELA, A. L.

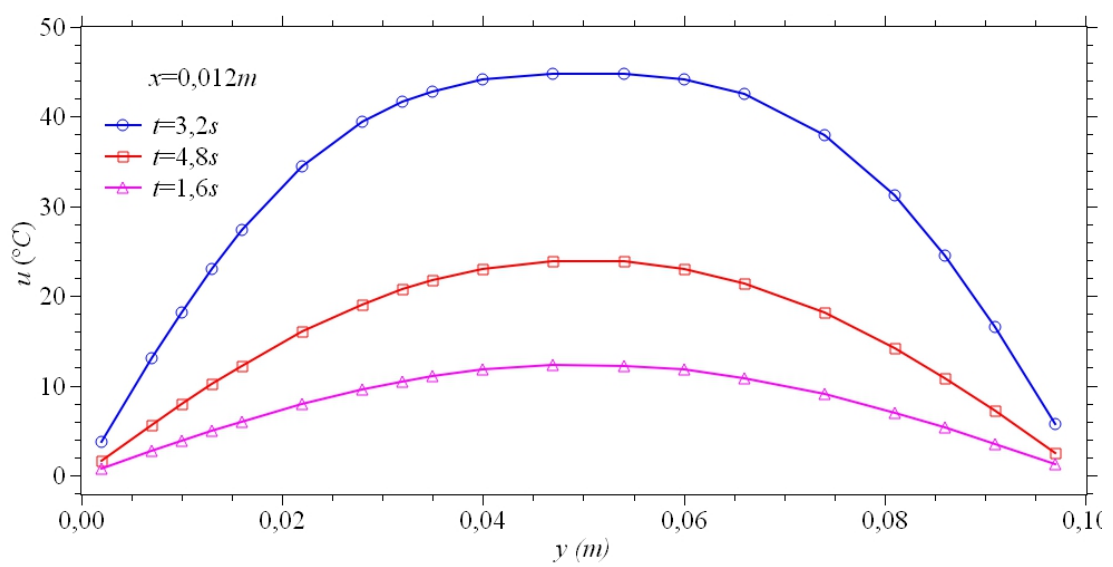

Figura 6. (Equação do calor bidimensional) Perfil de temperatura em função da coordenada $y$, para os tempos $t=1,6 s, t=3,2 s$ e $t=4,8 s$, fixando $x=0,012 m$. Fonte: Próprio autor.

Os efeitos físicos observados nas Figuras (4) e (5) podem ser vistos na Figura (7), que refere-se a representação gráfica do perfil de temperatura $u=u(x, y, t)$. A solução da equação do calor bidimensional, provinda do trabalho de [15], é equivalente a Equação (53).

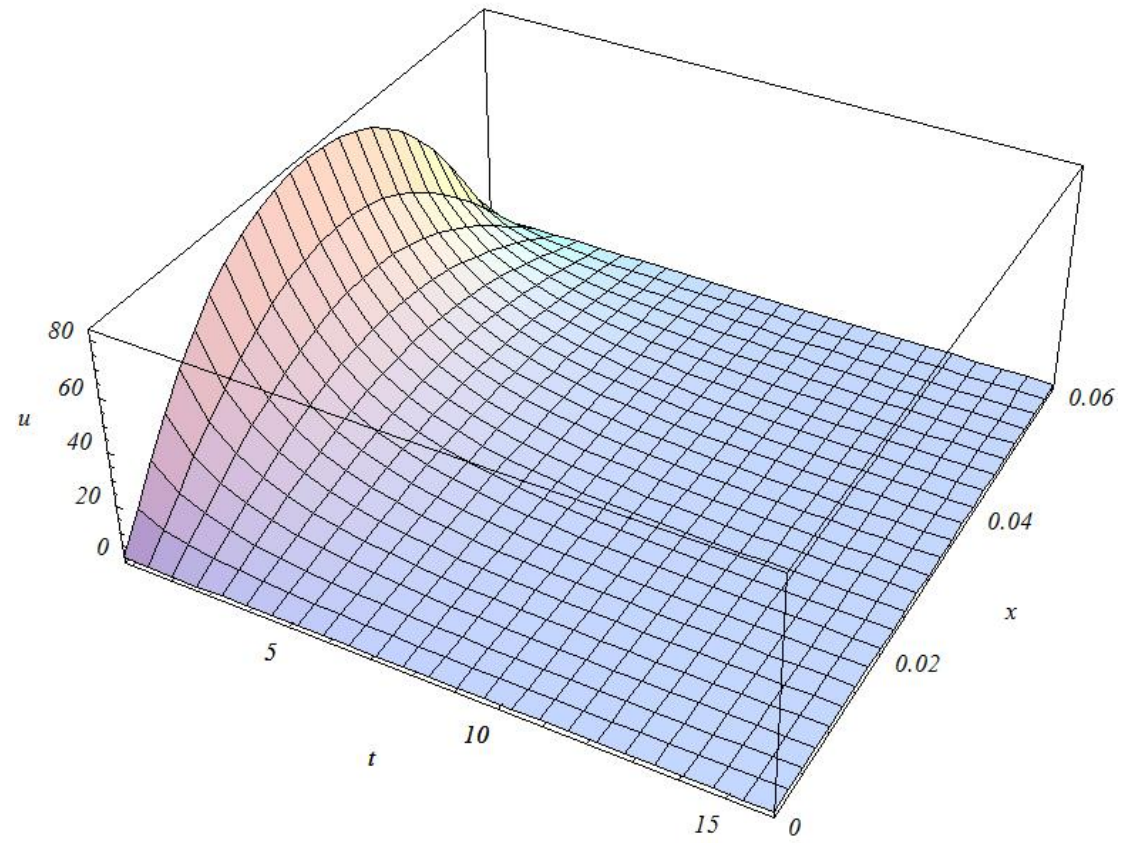

Figura 7. (Equação do calor bidimensional) Representação gráfica tridimensional do perfil de temperatura $u=u(x, y, t)$, considerando o domínio $(x, t) \in[0,00 ; 0,06] \times[0 ; 16]$, para $y=0,02 m$. Fonte: Próprio autor. 
Revista Ciências Exatas e Naturais, Vol.19, nº.2, Jul/Dez, 2017

\section{Conclusões}

Este trabalho teve como objetivo principal analisar a equação diferencial parcial do calor unidimensional e bidimensional, para determinar seu perfil através da resolução por meio da (TTIC) Técnica da Transformada Integral Clássica e Separação de Variáveis. Assim, com os resultados obtidos, pode-se verificar as temperaturas de acordo com o tempo em um condutor.

A aplicação da Técnica da Transformada Integral para a obtenção do campo de temperatura unidimensional e da Separação de Variáveis para o modelo bidimensional se mostraram ferramentas eficazes, pois, a partir dos resultados obtidos, vimos que o perfil de temperatura foi desenvolvido com grande êxito, em todas as condições de contorno impostas, isso pode ser comprovado após a análise dos gráficos e tabelas obtidos computacionalmente.

Como sugestão para futuros trabalhos, para dar continuidade a mesma linha de pesquisa, seria o desenvolvimento de um modelo bidimensional, para se observar como a temperatura se comporta e se haveria semelhança com os resultados obtidos a partir da construção de um experimento, que consiste no aquecimento das bordas em uma placa condutora. Dessa forma, pode-se comparar a solução analítica com resultados experimentais, isso seria um ponto importante a ser estudado.

\section{Referências}

[1] KARCZ, J; CUDAK, M; SZOPLIK, J. Stirring of a liquid in a stirred tank with an eccentrically located impeller. Chemical Engengineering Science, v.60, p. 2369-2380, 2005.

[2] SCHÄFER, M; KARASÖZEN, B; ULUDAG, Y; YAPICI, K; UGUR, Ö. Numerical method for optimizing stirrer configurations. Computational Chemical Engineering, v.30, p. 183-190, 2005.

[3] INCROPERA, F.P; DEWITT, D.P. Fundamentos de Transferência de Calor e de Massa. LTC, 3a Edição, Rio de Janeiro, 1990.

[4] AYDIN, O; AVCI, M. Analysis of laminar heat transfer in micro-Poiseuille flow. International Journal of Thermal Sciences, v. 46, p. 30-37, 2007.

[5] POVSTENKO, Y. Z. Fractional radial heat conduction in an infinite medium with a cylindrical cavity and associated thermal stresses. Mechanics Research Communications, v. 37, p. $436-440,2010$.

[6] D'AlESSAndRO, N. R. Estudo das Soluções Analíticas da Equação do Calor Unidimensional e Bidimensional. Dissertação de Mestrado - Universidade Federal de São Carlos, 2016.

[7] BRÉZIS, H. Análisis Funcional: Teoría y Aplicaciones. Alianza Editorial. Madrid, 1984.

[8] IÓRIO, V. EDP: Um Curso de Graduação. IMPA, Rio de Janeiro, 2016

[9] ÖZISIK, M.N. Heat Conduction. John Wiley, New York., 1980.

[10] CALLIOLI, C. A; DOMINGUES, H.H; COSTA, R.C.F. Álgebra Linear e Aplicações. 4a. edição, São Paulo, Atual, 1983. 
D'ALESSANDRO NETO, R. e VENEZUELA, A. L.

[11] QTIPLOT, SciDavis v. 1.D8. http://www.scidavis.sourceforge.net, 2014.

[12] DEVELOPERS, T. S: SageMath, the SageMathematics Software System (Version 7.2), http://www.sagemath.org, 2016.

[13] PÉRES, F, GRANGER, B. E. IPython: A System for Interactive Scientific Computing, Computing in Science and Engineering. Disponível em: http://ipython.org. Acessado em 27/04/2017. 2007.

[14] ZILL, D.G; CULLEN, M.R. Equações Diferenciais - Vol. 2. Pearson Makron Books, São Paulo, 2001.

[15] BIEZUNER, R.J. Equações Diferenciais Parciais Lineares. Disponível em: http://www.mat.ufmg.br/rodney. Acessado em 27/04/2017. 2010. 